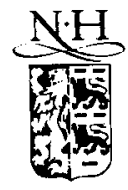

ELSEVIER

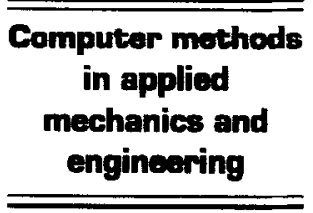

\title{
A high-order streamline Godunov scheme for steady supersonic flow computation
}

\author{
C.A. Hsu, J.Y. Yang * \\ Institute of Applied Mechanics, National Taiwan University, Taipei, Taiwan
}

Received 23 December 1992

\begin{abstract}
A space marching Godunov method using control volumes consisted of streamlines and the coordinate lines in the marching direction is described for the computation of two-dimensional and axisymmetric steady supersonic flows. These streamline meshes are solution-generated. They follow the fluid particles closely and also simplify the implementation of the Godunov method. A second order extension to the Godunov scheme using essentially nonoscillatory interpolation is also presented. Computations of various supersonic and hypersonic flow problems indicate that the method accurately represents smooth flow and crisply resolves all flow discontinuities such as shocks and slip lines even at high Mach numbers.
\end{abstract}

\section{Introduction}

The governing equations of motion for a compressible, inviscid, non-heat conducting fluid are the Euler equations. For supersonic flows, the steady Euler equations are hyperbolic and there exists a time-like variable which here is denoted as $x$ and characterized the space-marching direction. It is well known that there exists a mathematical analogy between two-dimensional steady supersonic flows and one-dimensional unsteady gasdynamic flows as both are belonged to the class of hyperbolic systems in two-independent variables. Physically, there is also a qualitative analogy between these two flows, namely oblique shocks, Prandtl-Meyer expansions and slip lines (tangential discontinuities) in the two-dimensional steady supersonic flows corresponding to the shock waves, rarefaction waves and contact discontinuities in one-dimensional unsteady gas dynamical flows. Because both systems are mathematically analogous they can be solved, in principle, using similar numerical procedures.

In the past few decades important advances have been made on the development of numerical methods for solving hyperbolic systems of partial differential equations in two independent variables, in particular, methods developed for solving the one-dimensional unsteady Euler equations of inviscid gas dynamics. Both the wave propagation property and conservation laws property have been unified to yield the well known upwind schemes for the computation of flows with shocks. For example, see recent review articles by Roe [1] and by Moretti [2]. Most of these methods are developed for the one-dimensional unsteady Euler equations but they should also be applicable to two-dimensional steady supersonic flows and allow one to solve the steady Euler equations using a space marching method. Indeed, the Godunov method [3] originally developed for 1-D unsteady Euler equations has been extended and applied by Glaz. and Wardlaw [4] to 2-D steady supersonic flows based

* Corresponding author. Tel: 886-2-363-0979; Fax: 886-2-363-9290; E-mail: yangjy@spring.iam.ntu.edu.tw. 
on the solution of the steady Riemann problem. Similarly, the random choice method $[5,6]$ has been applied by Marshall and Plohr [7] and the flux difference splitting method has been applied by Pandolfi [8] for the computations of steady supersonic flows. All these methods use the Eulerian description.

Recently, a class of new Lagrangian formulations for the Euler equations of gas dynamics using a Lagrangian time and a stream function as independent variables has been proposed by Hui et al. [9-12] as an alternative way to describe the equations governing inviscid compressible flows. Applications of the Godunov method and the random choice method to the generalized Lagrangian formulation for steady supersonic and hypersonic flow computations have been given in [10] and [12], respectively. This newly developed generalized Lagrangian method is noticeablc for its crisp resolution of slip lines in comparison to those resolved by using the Eulerian formulation. It is due to its intrinsic flow adaptive nature through the use of streamline and time lines as coordinate lines (also grid lines). In a steady supersonic flow slip lines are also streamlines and they coincide with each other thus resulting the perfect resolution of slip lines. It is also noted that the solution procedure of the steady Riemann problem is simplified considerably with the streamline meshes as control volumes or cells in the Godunov method.

In this paper, motivated by the flow adaptive nature of the generalized Lagrangian method, we study a space marching Godunov-type scheme with streamline meshes for steady supersonic flow computations using the steady Euler equations in Eulerian formulation. The control volumes or cells, composed of streamlines and constant $x$ lines, are constructed during each marching step. These strcamline meshes are expected to share the Lagrangian features of the adaptive flow nature and have the advantage of simplifying the implementation of the Godunov scheme. In general, during the construction of the cell interface flux in the Godunov method, the cell boundary may lie in any of the five regions separating the elementary waves in the Riemann solution or inside the Prandtl-Meyer expansion, and this complicates the programming somewhat. These procedures can be simplified with the streamline meshes in the present work. This is because the boundary between two adjacent cells always coincides with the slip line, which is also a streamline in steady flows, in the Riemann solution and this makes it easier to compute the flux there in the Godunov scheme. A second order extension to the Godunov method using a MUSCL-type approach [13] is also presented. The essentially nonoscillatory (ENO) interpolation of Harten and Osher [14] is used for the top and bottom states at the cell interfaces to achieve higher-order representations instead of piecewise constant states in the original Godunov method and produce high resolution nonoscillatory results.

In Section 2, the hyperbolic properties of the steady Euler equations of gas dynamics in supersonic flows are described. The elementary waves of the steady Riemann problem and its solution are given in Section 3 with emphasis on the streamline meshes. In Section 4, a Godunov-type scheme with streamline meshes for two-dimensional steady supersonic flows is presented. Extension of the first order Godunov scheme to a second order nonoscillatory scheme based on a new MUSCL-type approach is also described. In Section 5, numerical results for various steady supersonic and hypersonic flow problems are given to illustrate the salient feature of the present streamline Godunov method. Some concluding remarks are made in Section 6.

\section{Hyperbolicity of steady Euler equations in supersonic flows}

The Euler equations of motion of an inviscid non-heat conducting fluid for stcady two-dimensional and three-dimensional axisymmetric flows in conservation form can be written as

$$
\frac{\partial E}{\partial x}+\frac{\partial F}{\partial y}+G=0
$$

where

$$
E=\left(\begin{array}{c}
\rho u \\
\rho u^{2}+p \\
\rho u v \\
\rho u H
\end{array}\right)=\left(\begin{array}{c}
E_{1} \\
E_{2} \\
E_{3} \\
E_{4}
\end{array}\right), \quad F=\left(\begin{array}{c}
\rho v \\
\rho v u \\
\rho v^{2}+ \\
\rho v H
\end{array}\right), \quad G=K\left(\begin{array}{c}
\rho v / y \\
\rho u v / y \\
\rho v^{2} / y \\
\rho v H / y
\end{array}\right)
$$

Here, $x$ and $y$ are the physical coordinates, $u$ and $v$ are the corresponding components of the velocity, $\rho$ is the density, $p$ is the pressure, and $H$ is the total enthalpy per unit mass. The value of $K$ is 0 for the two-dimensional 
case and 1 for the axisymmetric one. In the last case, $y$ denotes the radial coordinate, and $x$ denotes the spacemarching direction in both cases. For a polytropic gas, the pressure is related to other fluid properties by the equation of state

$$
p=\frac{\gamma-1}{\gamma} \rho\left(H-\frac{1}{2} q^{2}\right)
$$

where $q^{2}=u^{2}+v^{2}$ and $\gamma$ is the ratio of specific heats.

The system of Eq. (1) can be put into the quasilinear form

$$
A \frac{\partial Q}{\partial x}+B \frac{\partial Q}{\partial y}+G=0
$$

with $Q=(\rho, u, v, p)^{T}$, the state variables of the fluid, and $A=\partial E / \partial Q$ and $B=\partial F / \partial Q$ are the Jacobian matrices of flux vectors $E$ and $F$. The conditions that ensure Eq. (4) is hyperbolic are that the determinant of $A$ is nonvanishing, i.e., det $A \neq 0$ and one can obtain real eigenvalues for the eigenvalue problem

$$
\operatorname{det}\left(A^{-1} B-\lambda_{i} I\right)=0
$$

Denoting $C=A^{-1} B$, Eq. (4) can be written as

$$
\frac{\partial Q}{\partial x}+C \frac{\partial Q}{\partial y}+A^{1} G=0
$$

The eigenvalues of $C$ are

$$
\begin{aligned}
& \lambda_{0}=\frac{v}{u} \quad \text { multiplicity of } 2 \\
& \lambda_{ \pm}=\frac{u v \pm a \sqrt{q^{2}-a^{2}}}{u^{2}-a^{2}}
\end{aligned}
$$

where $a=(\gamma p / \rho)^{1 / 2}$ is the speed of sound. To guarantee that matrix $C$ has 4 real eigenvalues, we require $q=\sqrt{u^{2}+v^{2}}>a$. That is, the flow must be supersonic. A characteristic whose slope is $\lambda_{0}$ (denoted as $C_{0}$ ) is a streamline while those with slopes $\lambda_{ \pm}$(denoted as $C_{ \pm}$) are Mach lines. Along characteristics, the fluid quantities are constrained by ordinary differential equations, i.e., the compatibility equations.

Along Mach lines $C_{ \pm}: \frac{d y}{d x}=\tan (\theta \pm \mu)$

$$
\frac{\mathrm{d} p}{\rho q^{2} \tan \mu} \pm \mathrm{d} \theta+K \frac{\sin \theta \sin \mu}{\sin (\theta \pm \mu)} \frac{\mathrm{d} y}{y}=0
$$

Along streamlines $C_{0}: \frac{\mathrm{d} y}{\mathrm{~d} x}=\tan \theta$

$$
\begin{aligned}
& \frac{\mathrm{d} \rho}{\mathrm{d} p}=\frac{1}{a^{2}} \\
& \frac{\mathrm{d} q^{2}}{\mathrm{~d} p}=-\frac{2}{\rho}
\end{aligned}
$$

where $\theta$ denotes the angle between the streamlines and the free stream direction $x$ and $\mu$ the local Mach angle $\sin ^{-1}(1 / M)$. The Mach number $M$ is defined by $M=q / a$.

For two-dimensional supersonic flows $(K=0)$ in a perfect gas, Eqs. (8) can be explicitly integrated to obtain the Riemann invariants

$$
\nu(M) \pm \theta=R_{ \pm} \quad \text { on } C_{ \pm} \text {characteristics }
$$

where $\nu(M)$ is the Prandtl-Meyer function defined by

$$
\nu(M)=\sqrt{\frac{\gamma+1}{\gamma-1}} \tan ^{-1} \sqrt{\frac{\gamma-1}{\gamma+1}\left(M^{2}-1\right)}-\tan ^{-1} \sqrt{M^{2}-1}
$$

It is well known that all small disturbances, except those of entropy and vorticity, are propagated along two of these families $C_{+}$and $C_{-}$; disturbances of entropy and vorticity are propagated along characteristic $C_{0}$, which coincides with the streamline. 


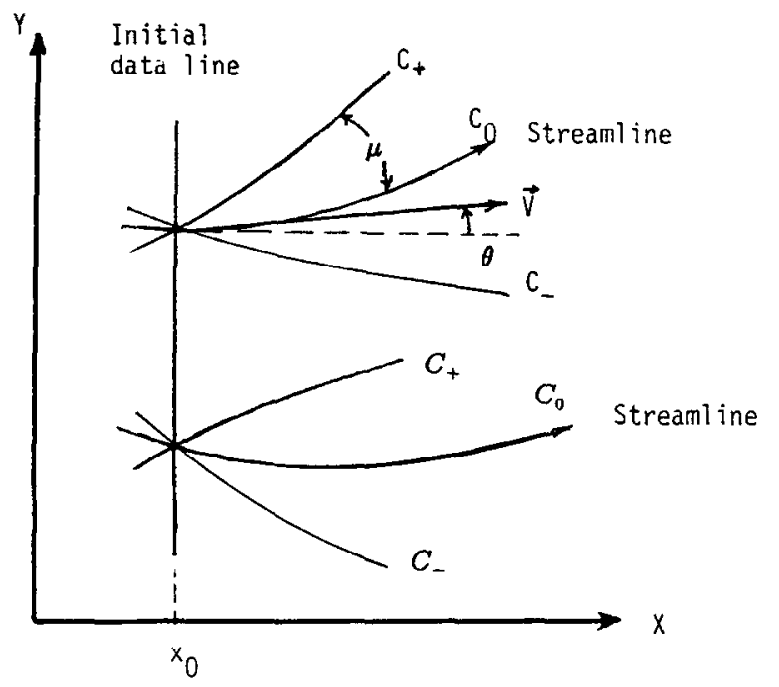

Fig. 1. Illustration of streamline, left- and right-running characteristic lines.

For a given supersonic flow, if the streamlines are known then the problem is to determine the characteristics belonging to the $C_{+}$and $C_{-}$families. If the streamlines can be used as coordinate lines, then the description of flow physics becomes rather simple and natural. One such example is the particularly elegant form of equations in natural coordinates. An illustration of streamlines and left- and right-running characteristics is sketched in Fig. 1. In the present work, we shall take advantage of this observation and using streamlines and constant coordinate $x$ lines as grid lines for the control volumes or cells used in the Godunov method. This will not only simplify the Godunov algorithm but also cnable the excellent resolution of slip lines without smearing.

\section{The Riemann problem of steady supersonic flow}

The Riemann problem for two-dimensional steady supersonic flow is specified as the system (1) of hyperbolic conservation laws subject to the initial condition

$$
Q\left(x=x_{0}, y\right)= \begin{cases}Q_{T}, & y>y_{0} \\ Q_{B}, & y \leqslant y_{0}\end{cases}
$$

where the flow states are given at the initial data line $x=x_{0}$ and $Q_{T}$ and $Q_{B}$ denote the top and bottom states.

The solution to the steady Riemann problem is self-similar in the variable $\left(y-y_{0}\right) /\left(x-x_{0}\right)$ and consists of three types of elementary waves, namely, the oblique shock wave $(s h)$, the Prandtl-Meyer expansion $(P-$ $M$ ) and the slip line (sl) separated by uniform regions in between (see Fig. 2). Denote the solution as $R\left[\left(y-y_{0}\right) /\left(x-x_{0}\right) ; Q_{B}, Q_{T}\right]$. These elementary waves can be used to construct solutions to the Riemann problem for steady two-dimensional supersonic flow. Let $Q_{0}$ and $Q$ be stats across one of the above elementary waves, then there are three cases:

(a) The wave is a slip line $\left(p=p_{0}\right)$.

In this case we have

$$
p=p_{0}=p^{*}, \quad \theta=\tan ^{-1}(v / u)=\theta_{0}=\tan ^{-1}\left(v_{0} / u_{0}\right)=\theta^{*}
$$

The pressure and flow angle must be identical on either side of the slip line. The density and tangential velocity components may jump abruptly.

(b) The wave is an oblique shock $\left(p>p_{0}\right)$

In this case we have, from the Rankine-Hugoniot oblique shock relation, for the flow direction angle,

$$
\Delta \theta= \pm \tan ^{-1}\left[\frac{\alpha-1}{\gamma M_{0}^{2}-\alpha+1}\left(\frac{2 \gamma M_{0}^{2}}{(\gamma+1) \alpha+(\gamma-1)}-1\right)^{1 / 2}\right] \text {, }
$$




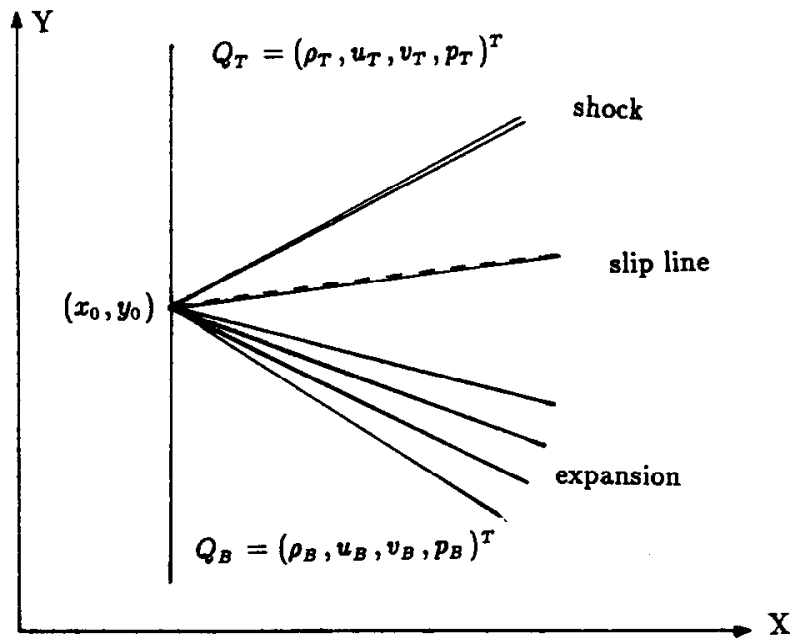

Fig. 2. Elementary waves in the solution of Riemann problem.

or the flow inclination

$$
\theta=\theta_{0} \pm \Delta \theta
$$

and

$$
\begin{aligned}
& \rho=\rho_{0} \frac{(\gamma+1) \alpha+\gamma-1}{(\gamma-1) \alpha+\gamma+1}, \\
& M=\left[\frac{M_{0}^{2}[(\gamma+1) \alpha+\gamma-1]-2\left(\alpha^{2}-1\right)}{\alpha[(\gamma-1) \alpha+(\gamma+1)]}\right]^{1 / 2} \\
& \alpha=p / p_{0}
\end{aligned}
$$

(c) The wave is a Prandtl-Meyer expansion $\left(p<p_{0}\right)$.

In this case we have

$$
M=\left[\frac{2}{\gamma-1}\left(\frac{1+(\gamma-1) M_{0}^{2} / 2}{\alpha^{(\gamma-1) / \gamma}}-1\right)\right]^{1 / 2}
$$

and

$$
\rho=\rho_{0} \alpha^{1 / \gamma} .
$$

The flow turning angle and inclination angle are respectively

$$
\Delta \theta=\mp\left[\nu(M)-\nu\left(M_{0}\right)\right], \quad \theta=\theta_{0}+\Delta \theta
$$

where $\nu(M)$ is the Prandtl-Meyer function defined in (10).

Therefore, through any state $Q_{0}$, with $\alpha=p / p_{0}$ as parameter, there are two families of states connecting to $Q_{0}$, namely, the compression state $(\alpha \geqslant 1)$, and the expansion state $(\alpha<1)$. The curves of two families have second order contact at $Q_{0}$ and can be regarded as one single family. This forms the basis of the Newton's method in the following solution procedure for solving the Riemann problem:

(i) In the $p-\theta$ plane there are two curves that pass through the states $Q_{0}=Q_{T}$ and $Q_{0}=Q_{B}$ they are defined respectively, by

$$
\theta=\Phi_{T}(\alpha)=\left\{\begin{array}{ll}
\theta_{T}+\tan ^{-1}\left[\frac{\alpha-1}{\gamma M_{T}^{2}-\alpha+1}\left(\frac{2 \gamma M_{T}^{2}}{(\gamma+1) \alpha+(\gamma-1)}-1\right)^{1 / 2}\right], & \alpha \geqslant 1, \\
\theta_{T}+\nu\left(M_{T}\right)-\nu(M), & \alpha<1
\end{array},\right.
$$

where $\alpha=p / p_{T}$, and

$$
\theta=\Phi_{B}(\alpha)=\left\{\begin{array}{ll}
\theta_{B}-\tan ^{-1}\left[\frac{\alpha-1}{\gamma M_{B}^{2}-\alpha+1}\left(\frac{2 \gamma M_{B}^{2}}{(\gamma+1) \alpha+(\gamma-1)}-1\right)^{1 / 2}\right], & \alpha \geqslant 1, \\
\theta_{B}-\nu\left(M_{B}\right)+\nu(M), & \alpha<1
\end{array},\right.
$$



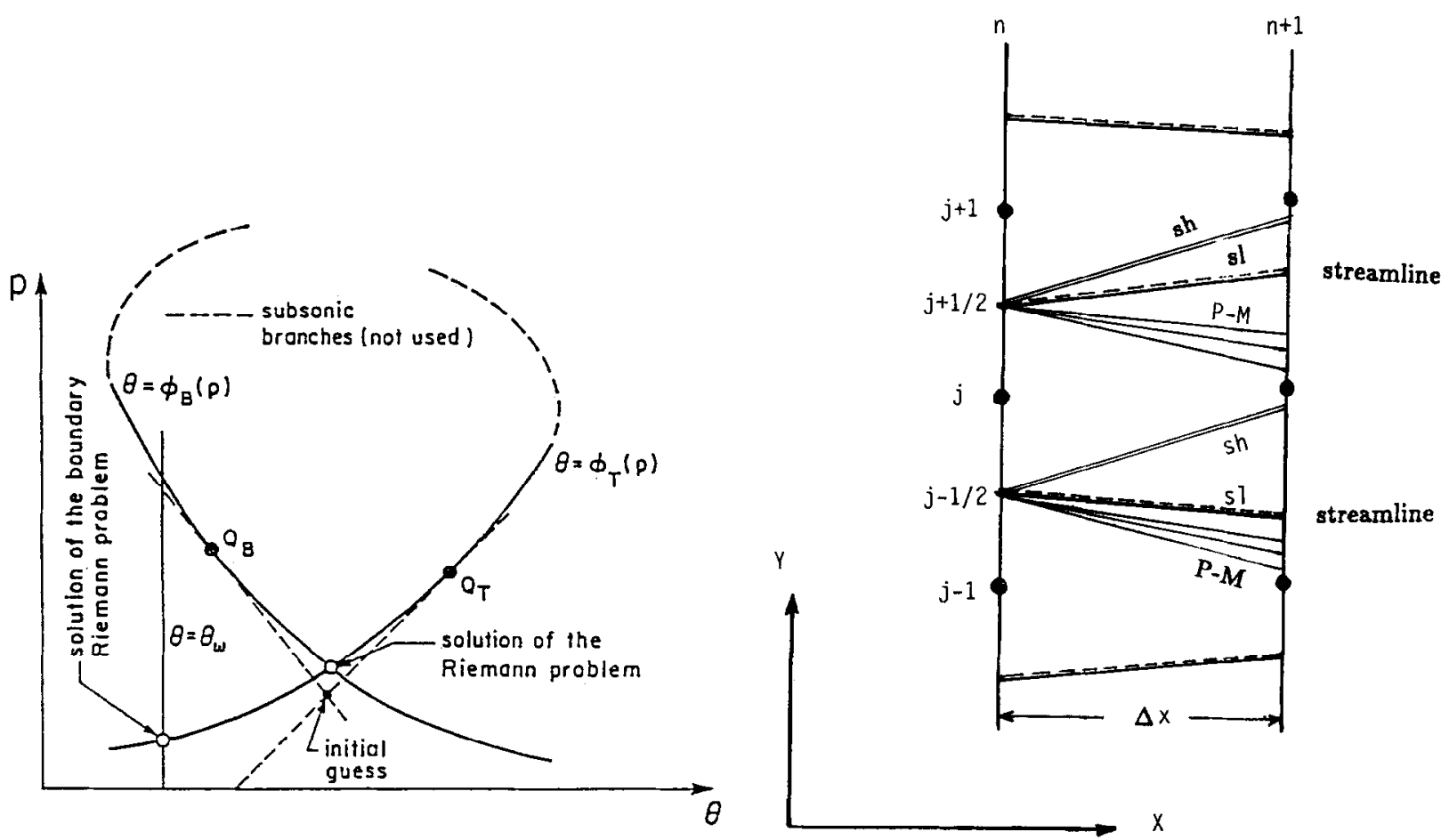

Fig. 3. Solution procedure of Riemann problem.

Fig. 4. Streamline meshes in $(x, y)$ coordinates.

where $\alpha=p / p_{B}$. These curves are depicted in Fig. 3.

Define function $f(p)$ as the difference between the flow angle behind a $(+)$-elementary wave and the flow angle behind a $(-)$-elementary wave.

$$
f(p)=\Phi_{T}\left(p / p_{T}\right)-\Phi_{B}\left(p / p_{B}\right)
$$

(ii) A standard Newton's iterative procedure is employed to find the intersect $\left(p^{*}, \theta^{*}\right)$ of the two curves.

The value of the pressure $p^{*}$ in the post $( \pm)$-elementary wave states is found by solving for the root of equation (23)

$$
f\left(p^{*}\right)=0 \text {. }
$$

The intersect of the two tangent lines passing through $Q_{T}$ and $Q_{B}$ is used as an initial guess to the solution. The exact derivatives are used here and usually it takes two to four iterations to converge to a tolerance $\epsilon \leqslant 10^{-6}$.

(iii) With the slip line values $p^{*}$ and $\theta^{*}$ known, we calculate $\alpha, \rho$ and $M$ from Eqs. (15) through (17), respectively, and hence also the flow speed $q=M \sqrt{\gamma p / \rho}$, for the uniform region between the slip line and the shock. For the uniform region between the slip line and the Prandtl-Meyer expansion we calculate $\alpha, \rho$ and $M$ from Eqs. (17), (19) and (18), respectively, and hence the flow speed $q=M \sqrt{\gamma p / \rho}$.

These flow quantities in the two uniform flow regions adjacent to the slip line are all that needed for computing the interface fluxes in the Godunov method.

At a solid wall the flow tangency condition requires that the solid wall is also a streamline. This causes a boundary Riemann problem and is specified as the system (1) of hyperbolic conservation laws subject to the condition

$$
Q\left(x=x_{0}, y\right)= \begin{cases}Q_{T}, & y>y_{w}, \\ Q_{B}, & y<y_{w}, . \\ \theta=\theta^{*}=\theta_{w}, & y=y_{w},\end{cases}
$$

Here, $Q_{B}$ is the mirror image flow of $Q_{T}$ with respect to $\theta_{w}$. In this way the tangency boundary condition is automatically satisfied. The solution procedure is then the same as that described above. 
An alternative way is to replace $\Phi_{B}$ with $\theta_{w}$ in equation (23) and solve for $p^{*}$ directly (see Fig. 3). The details are similar to that given in [10].

As mentioned before, the control volumes are made of streamlines, thus the flow inclination angle of each interface is known. This also causes a boundary Riemann problem and can be specified as the system (1) of hyperbolic conservation laws subject to the initial condition

$$
Q\left(x=x_{0}, y\right)= \begin{cases}Q_{T}, & y-y_{m}>\left(x-x_{0}\right) \tan \theta_{m} \\ Q_{B}, & y-y_{m} \leqslant\left(x-x_{0}\right) \tan \theta_{m}, \\ \theta=\theta_{m} & \end{cases}
$$

where $Q_{T}$ and $Q_{B}$ denote the constant states at the top and the bottom of the interface located at $y=$ $y_{m}+\left(x-x_{0}\right) \tan \theta_{m}$ and $\theta_{m}$ is the inclination angle of the interface and is equal to the average of $\theta_{B}$ and $\theta_{T}$. This is almost the same as the boundary Riemann problem for a solid wall except that the bottom state $Q_{B}$ is not the mirror image flow of $Q_{T}$. The solution procedure of boundary Reimann problem for (26) is the same as that for $(25)$.

The above elementary solutions of the Riemann problem will be used in the Godunov method to be described in the next Section and the Riemann solver can be also used to obtain the exact solution for some of the test problems.

\section{Godunov method using streamline meshes}

In this section, we describe the Godunov scheme [3] with streamline control volumes for the steady Euler equations in supersonic flows.

The computational mesh is illustrated in Fig. 4. The marching direction is $x$ and the mesh is oriented such that $x_{j}^{n}=$ constant $=x^{n}$ for all $j$. Here $n$ and $j$ refer to the marching step number and cell number, respectively. The marching distance, $\Delta x^{n}=x^{n+1}-x^{n}$, is chosen to satisfy the usual Courant-Friedrichs-Lewy (CFL) linear stability condition. The computational domain in $x-y$ plane is divided into a system of control volumes or cells which in the $y$-direction are centered at $y_{j}^{n}$ and have a height of $\Delta y_{j}^{n}=y_{j+1 / 2}^{n}-y_{j-1 / 2}^{n}$.

The difference equations for the $j$ th zone are obtained by integrating Eq. (1) (set $K=0$ temporarily) over the shaded region in Fig. 4 and applying the divergence theorem. Let $D$ and $\partial D$ denote the region and its boundary. Then,

$$
\iint_{D}\left(E_{x}+F_{y}\right) \mathrm{d} x \mathrm{~d} y=\int_{\partial D}(E, F) n \mathrm{~d} s=0,
$$

where $\boldsymbol{n}$ is the outward unit normal to $\partial D$. Expanding Eq. (27) and expressing in terms of a numerical flux, the difference equations become

$$
E_{j}^{n+1}=E_{j}^{n} \frac{\Delta y_{j}^{n}}{\Delta y_{j}^{n+1}}-\frac{\Delta x^{n}}{\Delta y_{j}^{n+1}}\left(G_{j+1 / 2}^{N}-G_{j-1 / 2}^{N}\right),
$$

where the numerical flux vector $G_{j+1 / 2}^{N}$ is defined as

$$
G_{j+1 / 2}^{N}=F_{j+1 / 2}^{n+1 / 2}-s_{j+1 / 2}^{n} E_{j+1 / 2}^{n+1 / 2}
$$

Here for any quantity $f$,

$$
f_{j}^{n}=\frac{1}{\Delta y_{j}^{n}} \int_{y_{j-1 / 2}^{n}}^{y_{j+1 / 2}^{n}} f\left(x^{n}, y\right) \mathrm{d} y,
$$

which is the cell-average of $f$ and

$$
f_{j+1 / 2}^{n+1 / 2}=\frac{1}{\Delta x^{n}} \int_{x^{n}}^{x^{n+1}} f\left(x, y_{j+1 / 2}^{n}+s_{j+1 / 2}^{n} \cdot\left(x-x^{n}\right)\right) \mathrm{d} x,
$$


where

$$
s_{j+1 / 2}^{n}=\left(y_{j+1 / 2}^{n+1}-y_{j+1 / 2}^{n}\right) / \Delta x^{n}
$$

In the first-order Godunov scheme the $j$ th cell average, $Q_{j}^{n}$, at marching step $n$ is considered as constant within that cell and the flux $F_{j+1 / 2}^{n+1 / 2}-s_{j+1 / 2}^{n} E_{j+1 / 2}^{n+1 / 2}$ along the interface between the $j$ th cell and the $(j+1)$ th cell from marching step $n$ to $n+1$ is to be obtained from the self-similar solution $R\left[\left(y-y_{j+1 / 2}^{n}\right) /\left(x-x_{j}^{n}\right) ; Q_{j}^{n}, Q_{j+1}^{n}\right]$ at $y=y_{j+1 / 2}^{n}$ to the Riemann problem formed by two adjacent piecewise constant flow states $Q_{j}^{n}$ and $Q_{j+1}^{n}$, i.e., with $Q_{j}^{n}=Q_{B}$ and $Q_{j+1}^{n}=Q_{r}$ and use the procedure described in Section 3.

The first order Godunov scheme given above is notably inaccurate in practice and needs to be upgraded to higher order accuracy. A second order Godunov-type scheme can be constructed for the supersonic steady Euler equations ( 1$)$ in the $(x, y)$ coordinates in a manner similar to van Leer's MUSCL approach [13]. Here, we present a new MUSCL-type second order scheme using an essentially nonoscillatory interpolation [14] to achieve high order accurate values of the cell interface.

The algorithm for computing the interface fluxes from the initial data, say, step $n$, and updating solutions to step $n+1$ may be divided into a number of steps. These are

Step 1. The "decoding" of $E_{j}^{n}$ to obtain $Q_{j}^{n}$. Let

$$
\chi^{2}=\gamma^{2}+\left(\gamma^{2}-1\right)\left(E_{3}^{2}-2 E_{1} E_{4}\right) / E_{2}^{2}
$$

Then

$$
\begin{aligned}
& u=\frac{\gamma+\chi}{\gamma+1} \frac{E_{2}}{E_{1}} \\
& v=E_{3} / E_{1}, \\
& \rho=E_{1} / u, \\
& p=E_{2}-u E_{1} .
\end{aligned}
$$

Step 2. Generation of streamline meshes. To start the calculation, we need grid points at step $n+1$ to build up computational cells. This may be done in several ways. In this work, the computational cell behaves locally like a fluid element and follows closely the fluid particle movement even when it crosses a shock where flow direction changes abruptly. These are carried out by defining

$$
\begin{aligned}
& x_{j}^{n+1}=x_{j}^{n}+\Delta x^{n} \\
& y_{j}^{n+1}=y_{j}^{n}+\Delta x^{n} \frac{v_{j}^{n}}{u_{j}^{n}}
\end{aligned}
$$

Here $\Delta x^{n}$ is the marching distance from step $n$ to step $n+1$ which is determined from the CFL condition based on the eigenvalues given in Eq. (7). And then, we can determine the cell height and the slope of the cell interface from

$$
s_{j+1 / 2}^{n}=\left(\frac{v_{j}^{n}}{u_{j}^{n}}+\frac{v_{j+1}^{n}}{u_{j+1}^{n}}\right) / 2 .
$$

Step 3. Second order accurate representation of values of cell interfaces. In this work, we first use essentially nonoscillatory (ENO) interpolation to get more accurate values at interfaces as

$$
\begin{aligned}
& E_{j+\frac{1}{2}}^{n-}=E_{j}^{n}+\frac{1}{2} \Psi_{j}^{n} \Delta y_{j}^{n}, \\
& E_{j-\frac{1}{2}}^{n+}=E_{j}^{n}-\frac{1}{2} \Psi_{j}^{n} \Delta y_{j}^{n},
\end{aligned}
$$

where

$$
\Psi_{j}^{n}=m\left(a_{j}^{n}, b_{j}^{n}\right)
$$

with 


$$
\begin{aligned}
& a_{j}^{n}=\frac{\Delta_{+} E_{j}^{n}}{\Delta_{+} y_{j}^{n}}-\zeta \Delta y_{j}^{n} \bar{m}\left(\frac{\Delta_{-} \Delta_{+} E_{j}^{n}}{\Delta_{-} \Delta_{+} y_{j}^{n}}, \frac{\Delta_{+} \Delta_{+} E_{j}^{n}}{\Delta_{+} \Delta_{+} y_{j}^{n}}\right), \\
& b_{j}^{n}=\frac{\Delta_{-} E_{j}^{n}}{\Delta_{-} y_{j}^{n}}+\zeta y_{j}^{n} \bar{m}\left(\frac{\Delta_{+} \Delta_{-} E_{j}^{n}}{\Delta_{+} \Delta_{-} y_{j}^{n}}, \frac{\Delta_{-} \Delta_{-} E_{j}^{n}}{\Delta_{-} \Delta_{-} y_{j}^{n}}\right) .
\end{aligned}
$$

Here $\Delta_{ \pm} E_{j}= \pm\left(\begin{array}{ll}E_{j \pm 1} & E_{j}\end{array}\right), \Delta_{ \pm} y_{j}^{n}= \pm\left(y_{j \pm 1}^{n}-y_{j}^{n}\right)$, denote the usual forward and backward difference operators. It is noted that the flux limiters are operating on flux difference $\Delta E_{j}$ type quantities.

The limiter functions $m$ and $\bar{m}$ are defined by

$$
m(a, b)= \begin{cases}\min (|a|,|b|), & \text { if } \operatorname{sgn} a=\operatorname{sgn} b=s \\ 0, & \text { otherwise. }\end{cases}
$$

and

$$
\bar{m}(a, b)= \begin{cases}a, & \text { if }|a| \leqslant|b| \\ b, & \text { if }|a|>|b|\end{cases}
$$

Then we advance $E_{j \neq 1 / 2}^{n \pm}$ to step $n+1 / 2$ as follows:

$$
\begin{aligned}
& \bar{E}_{j+\frac{1}{2}}^{-}=E_{j+\frac{1}{2}}^{n-}-\frac{\Delta x^{n}}{2 \Delta y_{j}^{n}}\left[F\left(E_{j+\frac{1}{2}}^{n-}\right)-F\left(E_{j-\frac{1}{2}}^{n+}\right)\right] \\
& \bar{E}_{j-\frac{1}{2}}^{+}=E_{j-\frac{1}{2}}^{n+}-\frac{\Delta x^{n}}{2 \Delta y_{j}^{n}}\left[F\left(E_{j+\frac{1}{2}}^{n-}\right)-F\left(E_{j-\frac{1}{2}}^{n+}\right)\right]
\end{aligned}
$$

If $\zeta=0$, one has a second order TVD scheme [15]; and if $\zeta=1 / 2$, one has a uniformly second order ENO scheme [14]. With $\bar{E}_{j \pm 1 / 2}^{\mp}$, one can decode it and obtain $\bar{Q}_{j \pm 1 / 2}^{\mp}$.

Step 4. Obtain the self-similar solution $R\left[\left(y-y_{j+1 / 2}^{n}\right) /\left(x-x_{j+1 / 2}^{n}\right) ; \bar{Q}_{j+1 / 2}^{+}, \bar{Q}_{j+1 / 2}^{-}\right]$by solving the Riemann problem.

The solution state is used to evaluate the interface fluxes $E_{j+1 / 2}^{n+1 / 2}$ and $F_{j+1 / 2}^{n+1 / 2}$ along $\left(y-y_{j+1 / 2}^{n}\right) /\left(x-x_{j+1 / 2}^{n}\right)=$ $s_{j+1 / 2}^{n}$. Finally, the solutions at step $n+1$ can be obtained from Eq. ( 28 ).

At this stage the numerical procedure is completed. To march forward further in $x$, one goes back to Step 1 and repeats Step 1-4.

In the case of axisymmetric flow, the splitting technique used for the quasi-one-dimensional unsteady flow is adopted as follows:

$$
E^{n+1}=L^{S}(\Delta x / 2) L^{E}(\Delta x) L^{S}(\Delta x / 2) E^{n}
$$

Here the operator $L^{S}$ represents the integration of source term, and the operator $L^{E}$ denotes the integration operation expressed in $\mathrm{Eq}$. (28). The $L^{S}$ operator is defined by

$$
L^{S}(\Delta x) E_{j}^{n}=E_{j}^{n}-\Delta x^{n} G\left(Q_{j}^{n}\right)+\frac{1}{2}\left(\Delta x^{n}\right)^{2}\left(\frac{\partial G}{\partial E}\right)_{j}^{n} G\left(Q_{j}^{n}\right)
$$

If a solid boundary is present in the flow, it must be a streamline. At the wall the velocity vector must be tangent to the wall. So the boundary condition to be imposed on the solid boundary is

$$
\frac{v}{u}=\tan \theta_{w}
$$

where $\theta_{w}$ is the inclination of the solid wall. This results in a boundary Riemann problem and is solved using the procedure described in Section 3 (see also [4,10]). As was demonstrated in references [4] and [10], this boundary treatment yields satisfactory results except in the presence of wall slope discontinuities. In those works, in order to improve the accuracy of the flow in the neighborhood of slope discontinuities a special treatment of numerical boundary condition is also described and implemented. In this paper, we take similar procedure except that no special treatment of wall slope discontinuities is used. 

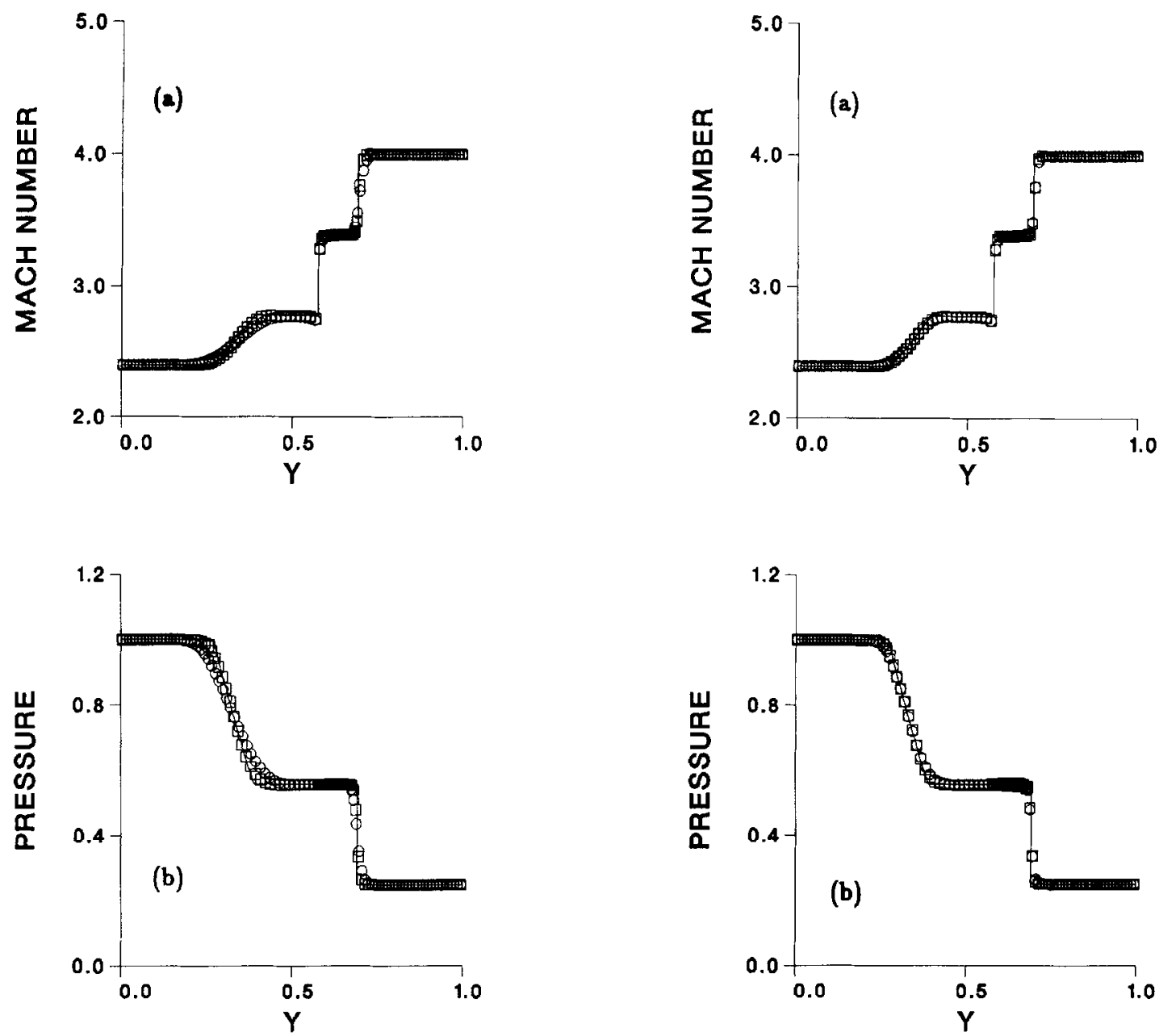

Fig. 5. Solution of stearly Riemann problem for supersonic flow. A comparison of first order (circles) and TVD (squares) results. (a) Mach number. (b) Pressure.

Fig. 6. Solution of steady Riemann problem for supersonic flow. A comparison of second order TVD (circles) and ENO (squares) results. (a) Mach number, (b) Pressure.

\section{Numerical results}

In this section, we first test the performance of the present second order nonoscillatory Godunov-type scheme with streamline meshes by solving several initial and boundary value problems of steady supersonic flows and comparing the results with the exact solutions. Computations of some practical supersonic and hypersonic flow problems are also included.

The first example is an initial value steady Riemann problem. It is formed by the confluence of two parallel supersonic streams at $y_{0}=0.5$ with different states. The initial conditions are given by

$$
Q= \begin{cases}Q_{T}=\left(\rho_{T}, p_{T}, M_{T}, \theta_{T}\right)=\left(0.5,0.25,4.0,0.0^{\circ}\right), & y>y_{0}, \\ Q_{B}=\left(\rho_{B}, p_{B}, M_{B}, \theta_{B}\right)=\left(1.0,1.0,2.4,0.0^{\circ}\right), & y \leqslant y_{0} .\end{cases}
$$

This is a case considered by Glaz and Wardlaw [4]. In the exact solution, the resulting interaction produces an oblique shock wave, a Prandtl-Meyer expansion and a slipline in between.

Here we use 100 cells and $\Delta y_{\text {init }}=0.01$. Numerical results for the flow properties at section $A-A^{\prime}(x=0.5)$ are shown in Fig. 5 along with the exact solution (solid line) which was obtained using the Riemann solver. Results obtained using both first order Godunov scheme (circles) and second order TVD scheme (squares) 


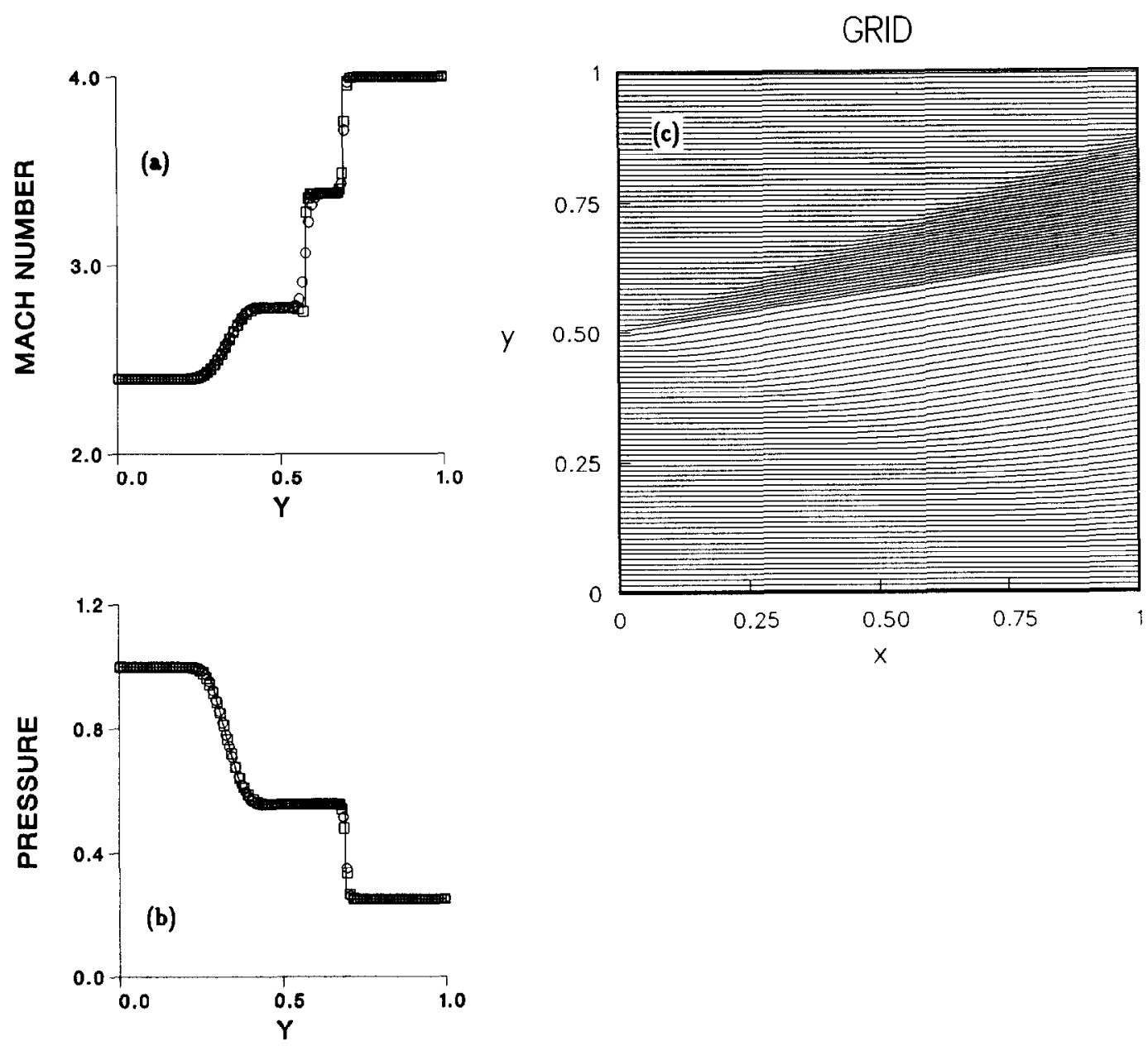

Fig. 7. Solution of steady Riemann problem for supersonic flow. A comparison of Cartesian mesh (circles) and streamline mesh (squares) results. (a) Mach number. (b) Pressure. (c) Streamline meshes.

with $\mathrm{CFL}=0.95$ are shown. All computations are done on a Convex $\mathrm{C} 1$ vector computer. The CPU time is 2.33 seconds for the first order scheme and 2.55 seconds for the second order TVD scheme. A comparison of results using second order TVD and ENO schemes with CFL $=0.95$ are shown in Fig. 6. The ENO results (squares) indicate slightly better resolution at shocks and Prandtl-Meyer expansions. The CPU time for the second order ENO scheme is 2.79 seconds. A comparison of computations using streamline meshes and rectangular Cartesian meshes, respectively, is given in Fig. 7 using the second order TVD scheme. Both mesh systems indicate good resolutions of shocks, but the streamline mesh system has much better resolution of slip line. This may be attributed to the streamline meshes, also shown in Fig. 7(c), which follow the movement of the fluid particles. To test the robustness of the method, a more severe case with $M_{T}=12$ is considered. The results are shown in Fig. 8 at section $A-A^{\prime}(x=0.8)$ using the second order TVD scheme (circles) with CFL $=0.95$ together with the exact solution (solid lines). Again we use 100 cells and $\Delta y_{\text {init }}=0.01$. It is observed that the quality of resolution for shock and slip line remain unchanged even with increasing Mach number. This is another feature of using streamline meshes as computational cells.

Secondly, in order to further examine the performance of the present second-order nonoscillatory schemes we consider a supersonic channel flow as an internal boundary problem. The channel has a straight upper wall and a 5\% thick circular arc bump on the lower wall. A comparison of results using second order ENO and first order Godunov schemes with 50 cells, $\Delta y_{\text {init }}=0.01$ and CFL $=0.9$ is shown in Fig. 9. Here only the pressure contours and grid lines are shown. The ENO results indicate much better resolution of leading edge shock, reflection shocks, trailing edge shock and the resulting shock-shock interactions. It is interesting and unexpected to find that the ENO scheme spend slightly fewer CPU time in this case. The CPU time is 9.3 and 

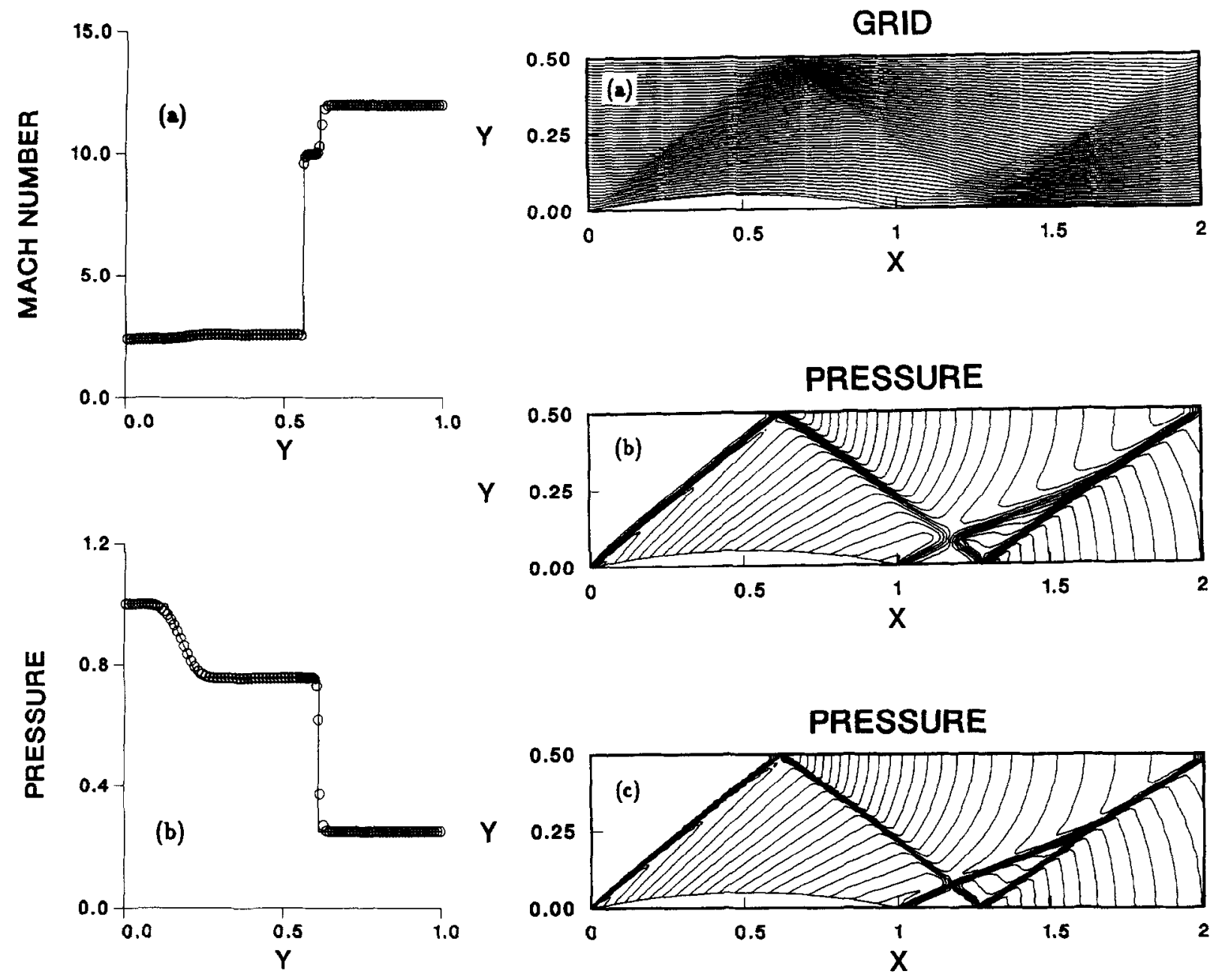

Fig. 8. Solution of steady Riemann problem for hypersonic flow. (a) Mach number. (b) Pressure.

Fig. 9. Supersonic flow in a channel with $5 \%$ thick circular arc bump, $M_{\infty}=2.0$. (a) Grid lines. (b) First order Godunov scheme. (c) Second order ENO scheme.

9.8 seconds for ENO scheme and Godunov scheme, respectively.

Thirdly, we consider a supersonic flow $M_{\infty}>1$ past a concave wall for which a shock wave of finite strength is formed suddenly in the interior of the flow field and consequently slip line is also generated. The equation for such a profile is given in [10].

As an example we consider the case when $M_{\infty}=4.0$ and $M_{1}=2.867$. The flow field is computed using the second order TVD scheme with 80 cells and $\Delta y_{\text {init }}=0.01$. In Fig. 10, the computed pressure contours, density contours, Mach number contours, and entropy contours are shown. The sudden birth of an oblique shock wave of finite strength in the interior of the flow field and the accompanying Prandtl-Meyer expansion and the slip line are seen well-captured. The distributions of flow properties along a constant distance line $(x=1.15)$ are shown in Fig. 11 together with the exact solutions (solid lines).

We also consider supersonic flows over two-dimensional profiles. Two type of profiles are considered. One is a $10 \%$ thick $(t / c=0.1)$ symmetric circular-arc airfoil and the other is a symmetric double-wedge airfoil with wedge angle $10^{\circ}$. For these two airfoils, 200 uniform cells with $\Delta y_{\text {init }}=0.01$ are used and CFL $=0.9$. In Table 1, the computed lift coefficient $C_{l}$ and wave drag coefficient $C_{d}$ (top line) for supersonic flow over a symmetric circular arc airfoil $(t / c=10 \%)$ with several angles of attack are listed together with results obtained using shock expansion theory (middle line) and linearized theory (bottom line). Excellent agreement is found between the present computation and the shock expansion theory. Results from the linearized theory deviate 

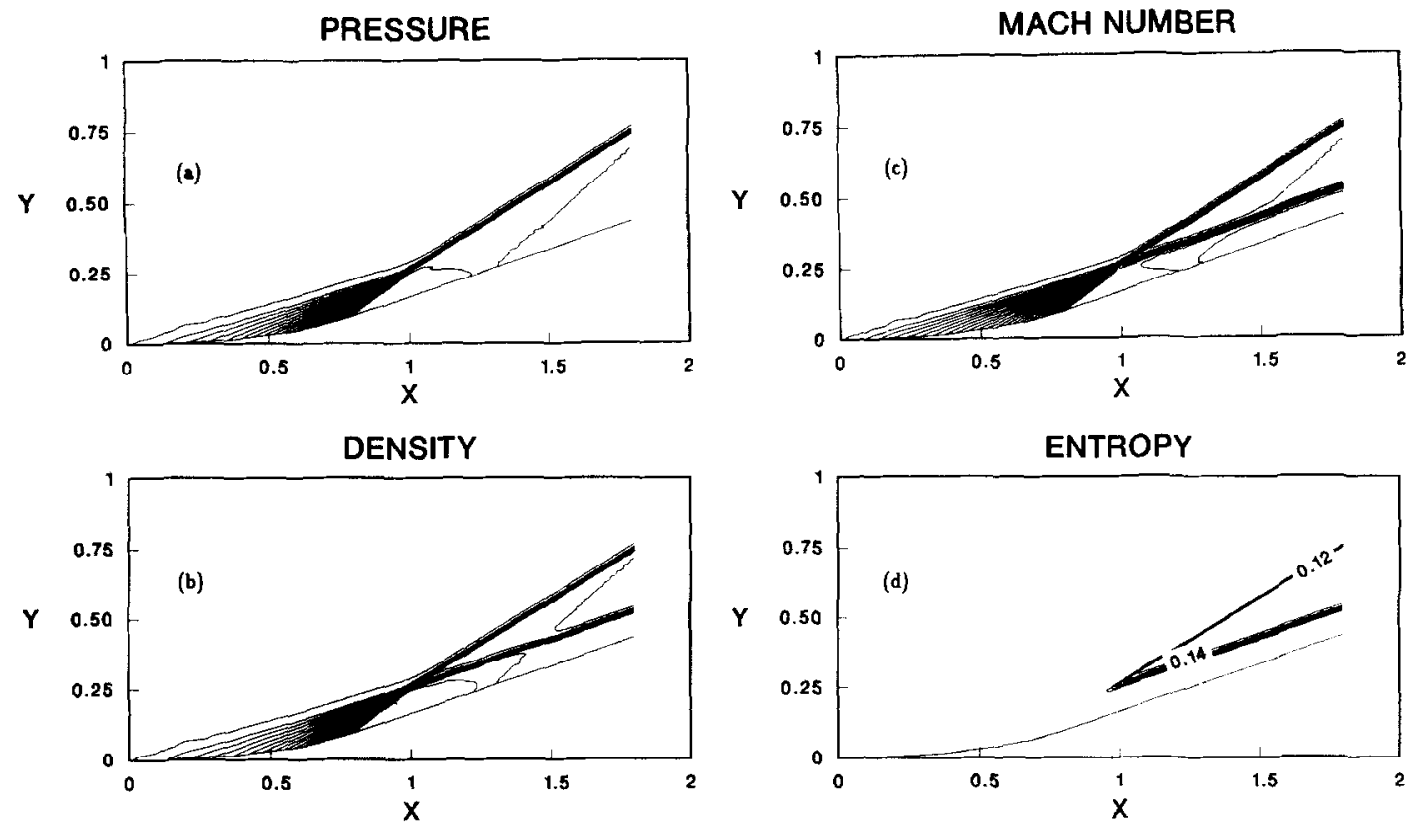

Fig. 10. Sudden formation of oblique shock wave of finite strength ( $M_{\infty}=4.0, M_{1}=2.867$ ). Second-order TVD solution. (a) Pressure contours. (b) Density contours. (c) Mach number contours. (d) Entropy contours.

Table 1

Comparison of the aerodynamic parameters for the two-dimensional circular-arc airfoil with $M_{\infty}=2.0$ and $t / c=0.1$

\begin{tabular}{cll|lll}
\hline$\alpha$ & $C_{l}$ & $C_{d}$ & $\alpha$ & $C_{l}$ & $C_{d}$ \\
\hline $0^{\circ}$ & 0.000003 & $0.031127^{\mathrm{a}}$ & $6^{\circ}$ & 0.252057 & 0.058771 \\
& 0.000000 & $0.031229^{\mathrm{b}}$ & & 0.253700 & 0.058980 \\
& 0.000000 & $0.030916^{\mathrm{c}}$ & & 0.241840 & 0.056241 \\
$2^{\circ}$ & 0.083513 & 0.034148 & $8^{\circ}$ & 0.338133 & 0.080762 \\
& 0.083908 & 0.034274 & & 0.340716 & 0.081155 \\
& 0.080613 & 0.033730 & & 0.322453 & 0.075939 \\
$4^{\circ}$ & 0.167354 & 0.043360 & $10^{\circ}$ & 0.426303 & 0.109878 \\
& 0.168297 & 0.043464 & & 0.429280 & 0.110426 \\
& 0.161227 & 0.042172 & & 0.403067 & 0.101264 \\
\hline
\end{tabular}

${ }^{\mathrm{a}}$ Present computation. ${ }^{\mathrm{b}}$ Shock expansion theory. ${ }^{\mathrm{c}}$ Linearized theory.

substantially from those of the nonlinear theory and the Euler computation at increasing angle of attack.

In Fig. 12, the flow fields around a symmetric circular-arc airfoil in a supersonic flow with $M_{\infty}=3.0$ and $0^{\circ}$ angle of attack are shown. The leading edge and trailing edge oblique shock waves and the Mach waves emanating from the surface are all crisply resolved. The grid lines clearly indicate that the computational mesh follows exactly the particle movement even when it crosses a shock wave. The CPU time after marching a chord length behind the trailing edge is 27.3 seconds. The computed pressure contours and particle traces (grid lines) are given in Fig. 13 for hypersonic flow with $M_{\infty}=8.0$ and $0^{\circ}$ angle of attack. In this case, the initial mesh distribution is stretched with $\Delta y_{\min }=0.001$. Again, sharp and clean representations of the flow fields are illustrated. The second order TVD scheme is used for both cases and CFL $=0.9$.

Computed results for supersonic flow past a symmetric double-wedge airfoil with $10^{\circ}$ wedge angle are given in Fig. 14 for freestream Mach number $M_{\infty}=3.0$ and $0^{\circ}$ angle of attack using second order TVD scheme. The leading edge oblique shocks, the Prandtl-Meyer expansion fans and the trailing edge oblique shocks are all crisply represented, and the flow adaptive feature is easily illuminated via the streamlines (grid lines). The CPU time for this case is 40 seconds.

Another example is a supersonic flow $M_{\infty}=2.05$ past a $10 \%$ symmetric circular arc profile in a channel with $4^{\circ}$ angle of attack. A shadowgraph taken from Ref. [16] is used for comparison. The pressure contours, density contours and the resulting particle traces (grid lines) are shown in Fig. 15 obtained using the second order TVD scheme. The number of cells used is 180 with $\Delta y_{\text {init }}=0.01$ and CFI $=0.9$. Good agreement is observed 

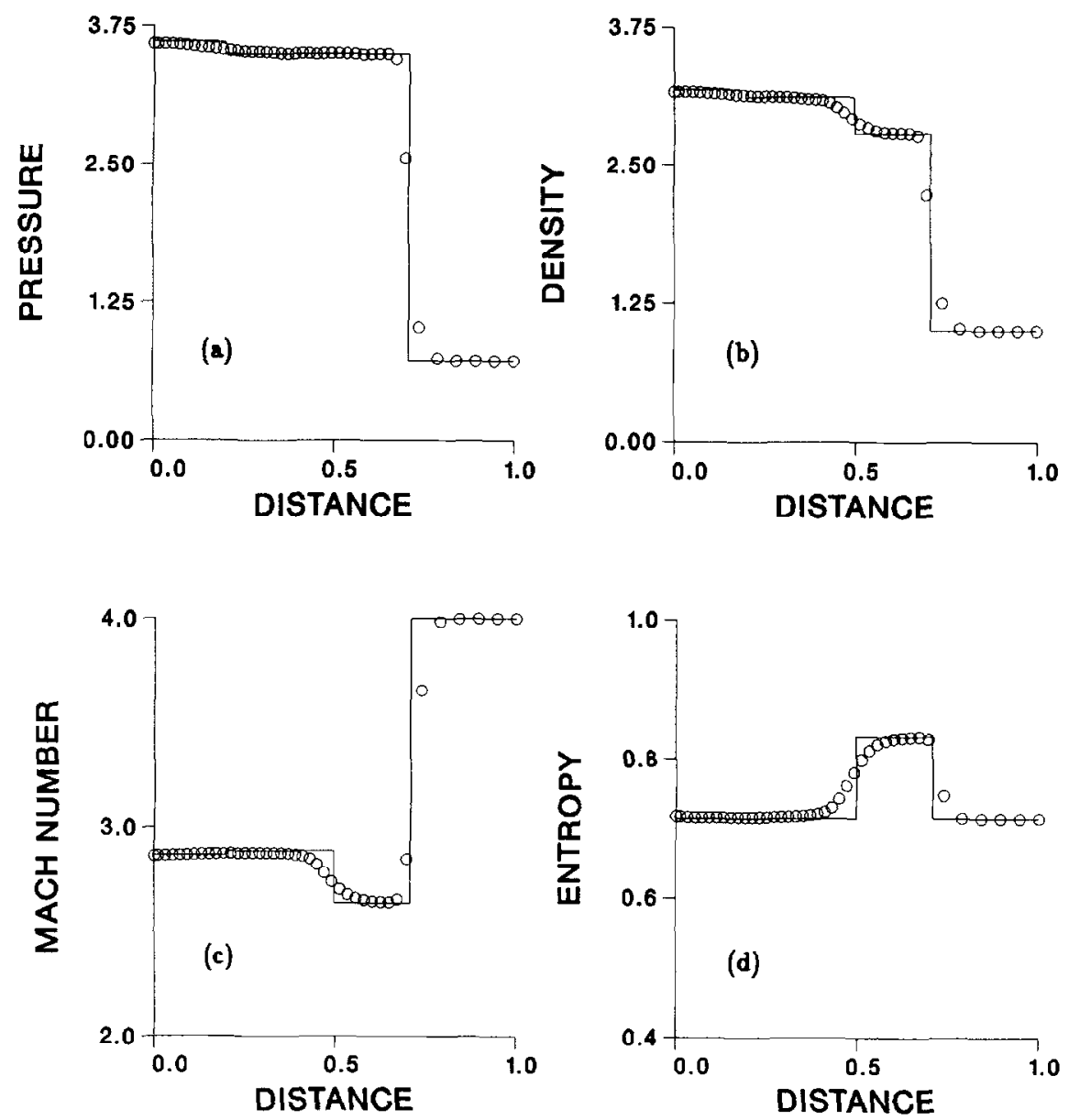

Fig. 11. Distributions of flow properties along a marching distance line $(x=1.15)$. (a) Pressure. (b) Density. (c) Mach number. (d) Entropy.

in nearly every aspect except regions due to viscous effects such as the shock/boundary layer interaction at walls and the wake flow at the trailing edge.

The next example is an experimental case on supersonic flow with $M_{\infty}=1.87$ past a concave wall shape consisted of two circular-arc profiles used by Johannesen [17]. In this case, there is a surface discontinuity at the junction of the two circular arcs. The computed pressure contours, Mach number contours and a Schlieren photograph taken from Ref. [17] are shown in Fig. 16. The number of cells used is 125 with $\Delta y_{\text {init }}=0.01$ and $\mathrm{CFL}=0.9$. Good agreement is found between the computational and experimental results.

The last example we consider is supersonic flow with $M_{\infty}=2.0$ past an axisymmetric circular cone with $20^{\circ}$ cone angle. Here we use 70 cells and $\Delta y_{\text {init }}=0.01$ and $\mathrm{CFL}=0.90$. Computed results for the flow properties at section $A-A^{\prime}(x=0.6)$ are shown in Fig. 17 along with the exact solution (solid line) which was obtained by solving the Taylor-Maccoll equation. Very good agreement is found between the computation and exact solution.

\section{Concluding remarks}

In this paper, a Godunov method with streamline meshes as control volumes is presented for solving the steady Euler equations of supersonic gas dynamics. A second order accurate scheme using essentially nonoscillatory interpolation is also developed. Computations of various two-dimensional and three-dimensional axisymmetric steady supersonic and hypersonic flow problems have been carried out and the results indicate that the smooth flow can be accurately represented and flow discontinuities such as oblique shock waves and slip lines can 

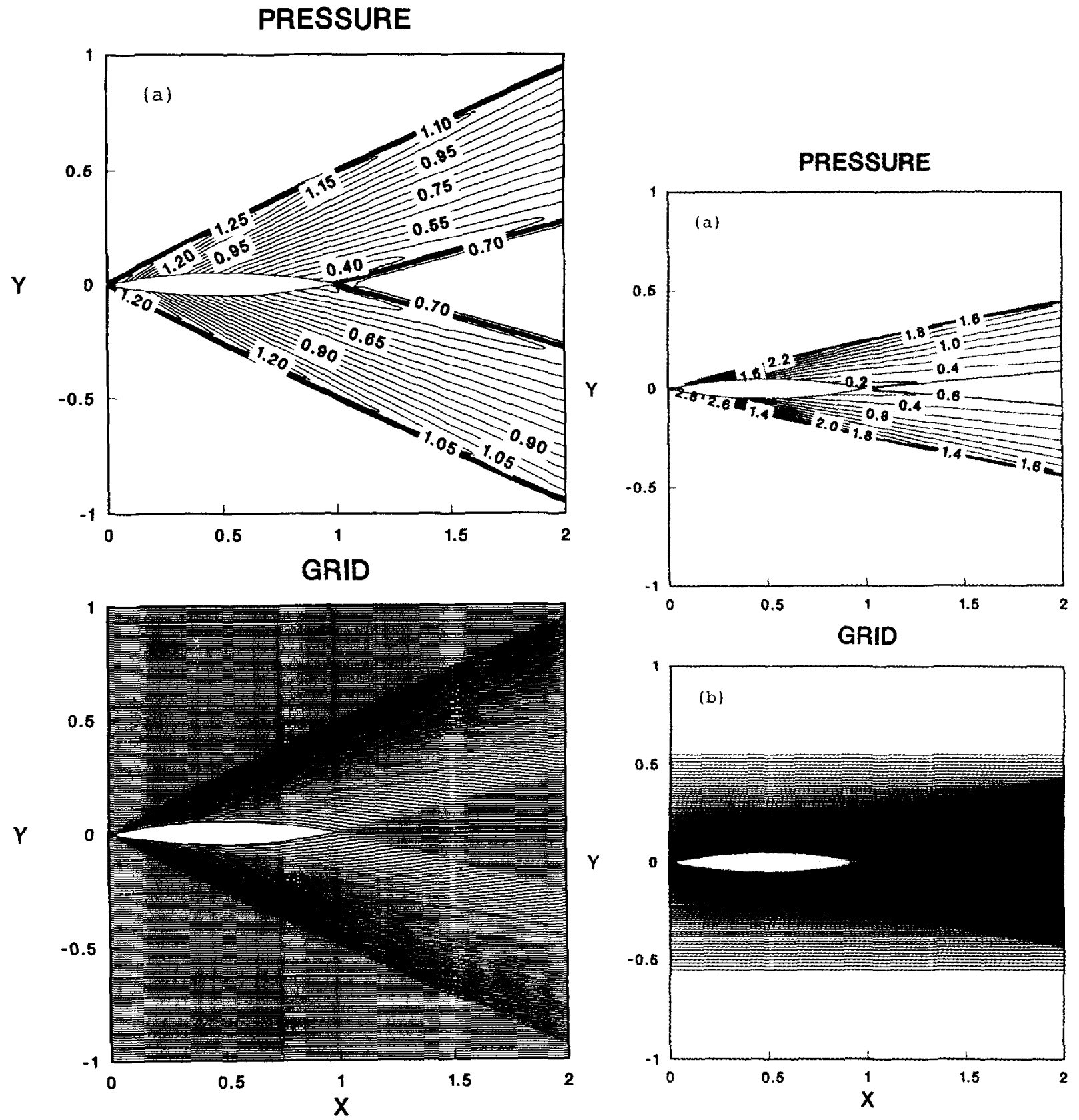

Fig. 12. Steady supersonic flow around a symmetric circular arc airfoil $\left(M_{\infty}=3.0, \alpha=0^{\circ}\right)$. (a) Pressure contours. (b) Particle traces (grid lines).

Fig. 13. Steady hypersonic flow around a sysmetric circular arc airfoil $\left(M_{\infty}=8.0, \alpha=0^{\circ}\right)$. (a) Pressure contours. (b) Particle traces (grid lines).

be crisply resolved. The quality of results remain the same even at high Mach number. The main features of the present method are first its use of the streamline meshes as computational cells, with the consequence that intrinsic flow adaption is embedded and the body surface boundary condition is automatically satisfied. Second, the slip line is also a streamline and always coincides with the cell interface which renders not only excellent resolution of slip lines but also simple implementation of the Godunov method. Thus the present method shares the same desirable features of the new Lagrangian method of Hui and Loh [10,11] but with a Eulerian formulation. 

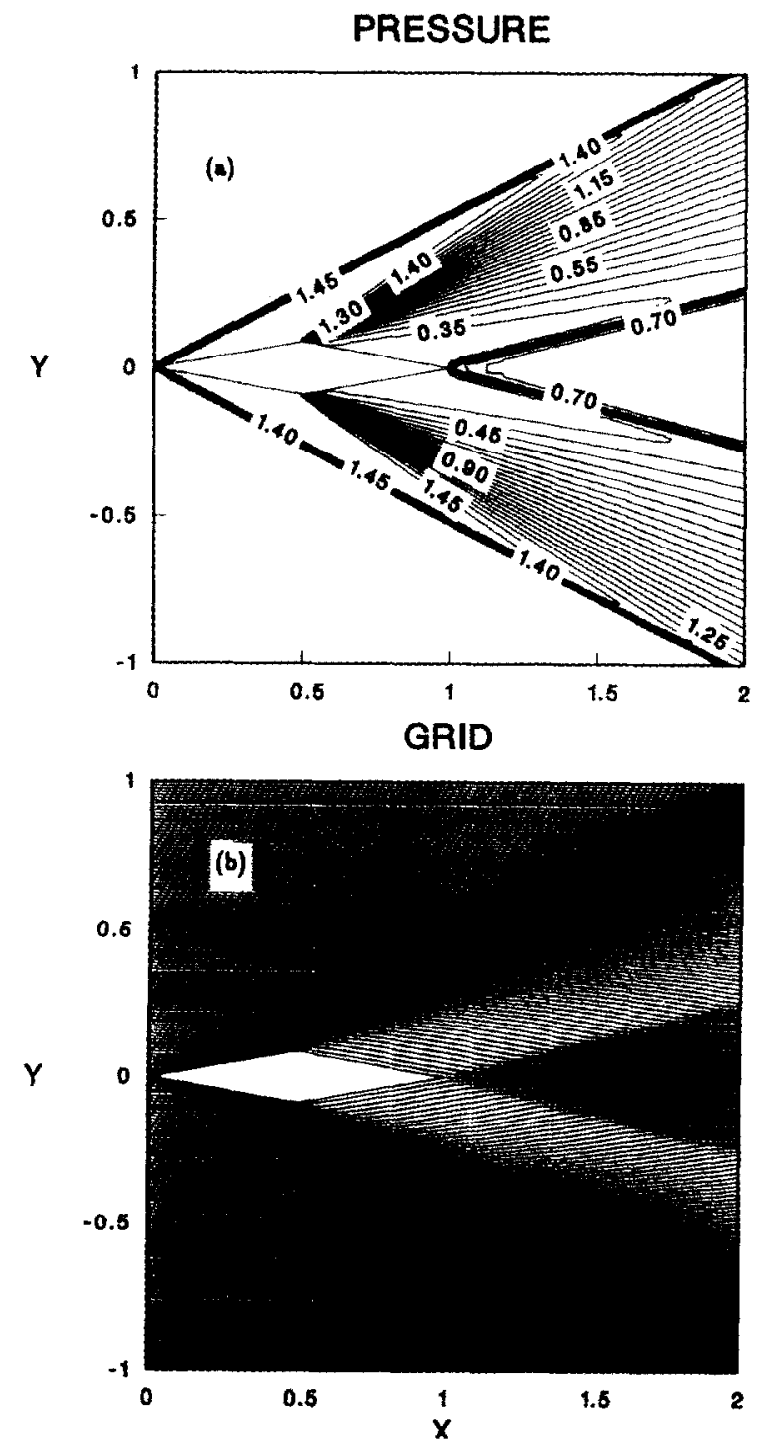

Fig. 14. Steady supersonic flow around a double-wedge airfoil $\left(M_{\infty}=3.0, \alpha=0^{\circ}\right)$. (a) Pressure contours. (b) Particle traces (grid lines).

Several supersonic and hypersonic flows are computed and compared with exact solutions and available experimental data. Very good agreement among the computational, theoretical and experimental results have been obtained. The computational algorithm is fast, robust and accurate.

Extension of the present streamline Godunov method to three-dimensional case can be done in a similar way as $[18]$.

\section{Acknowledgement}

This research was supported by the National Science Council of the Republic of China under contract NSC 81-0210-E004-49. 

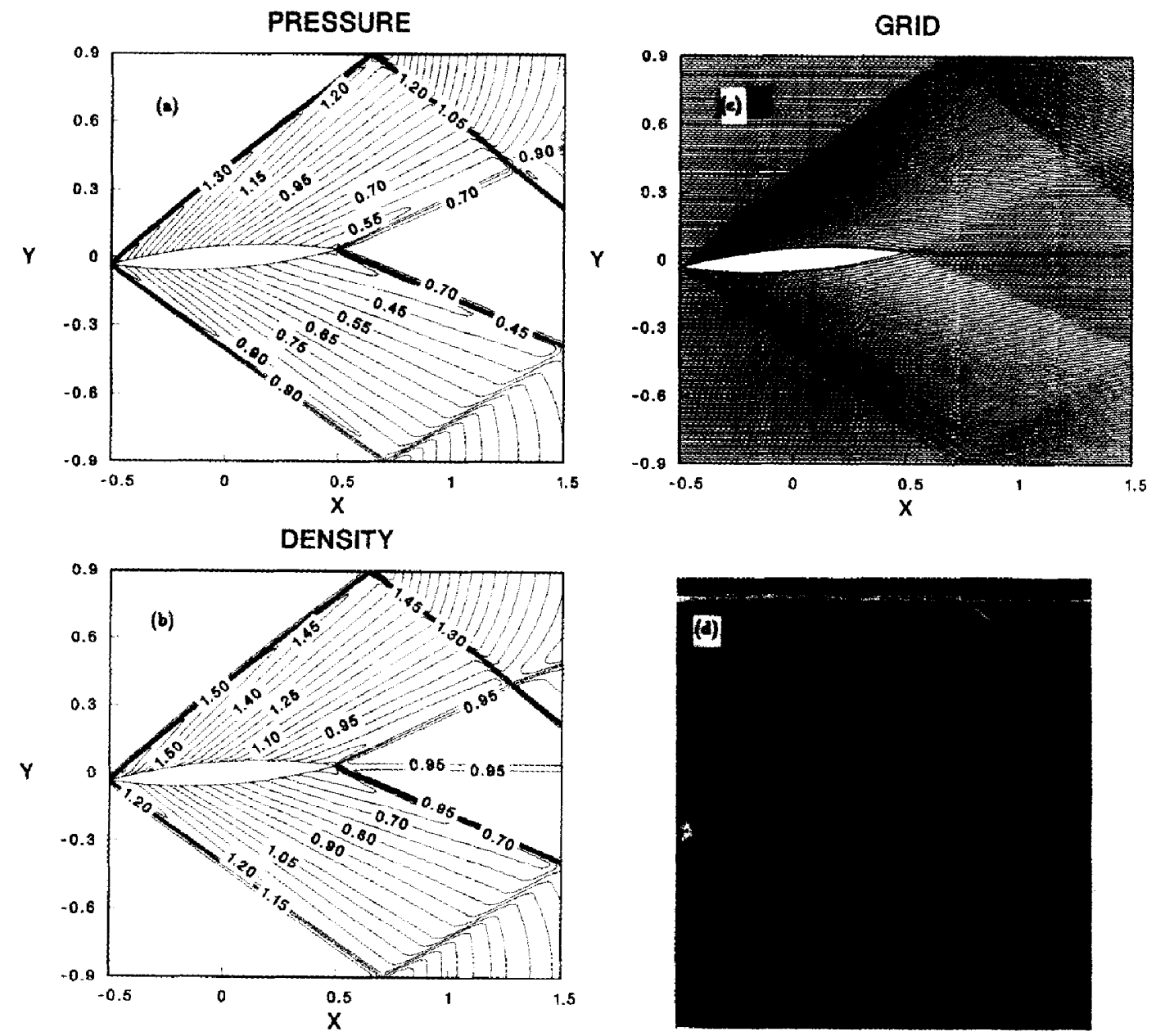

Fig. 15. Supersonic flow around a symmetric circular arc airfoil ( $\left.M_{\infty}=2.05, \alpha=4^{\circ}\right)$. TVD solution. (a) Pressure contours. (b) Density contours. (c) Particle traces. (d) Experimental shadowgraph (Ref.[16]). 

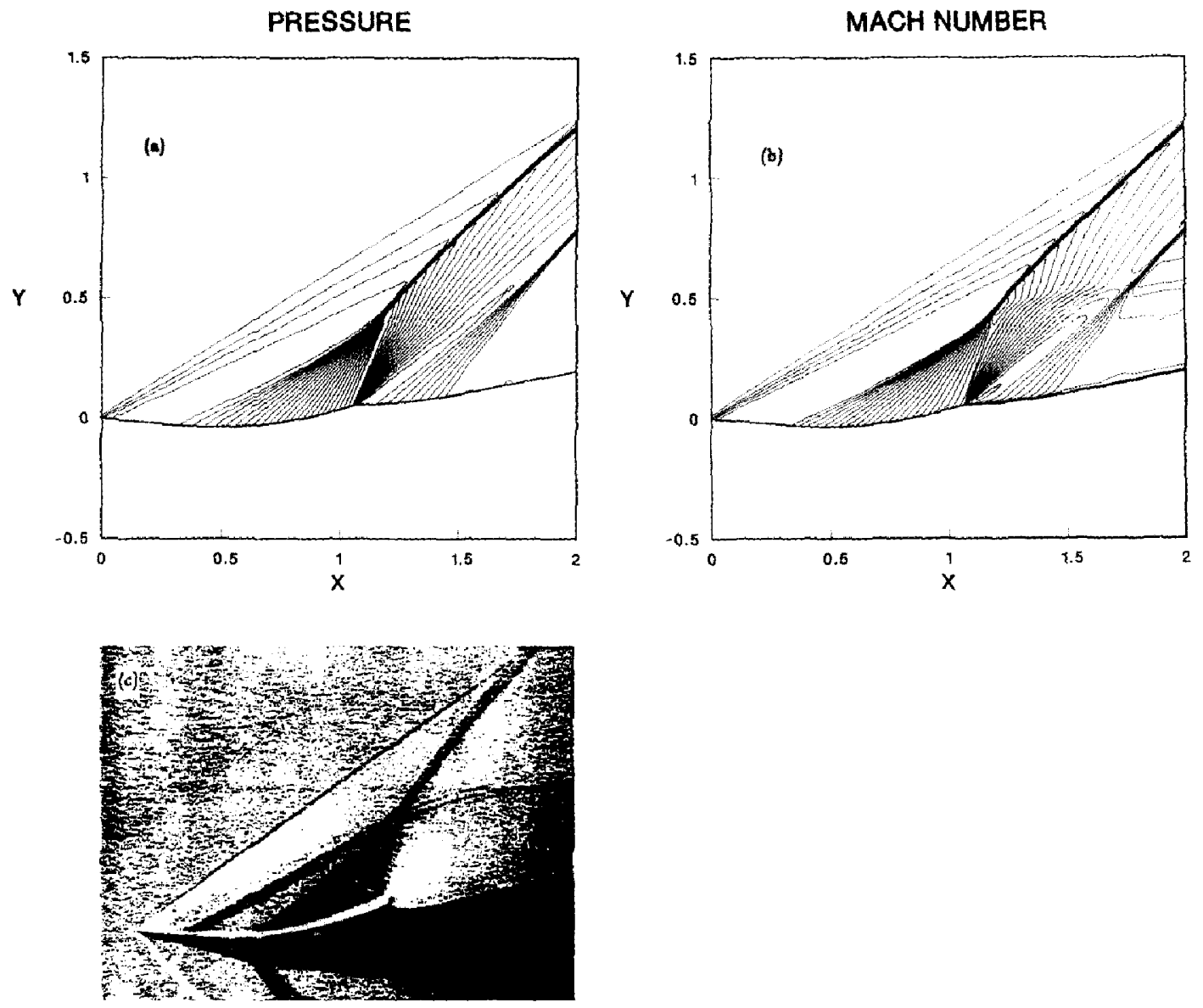

Fig. 16. Supersonic flow around a concave profile $\left(M_{\infty}=1.87\right.$ ). (a) Pressure contours. (b) Mach number contours. (c) Experimental Schlieren photograph (Ref. [17]). 

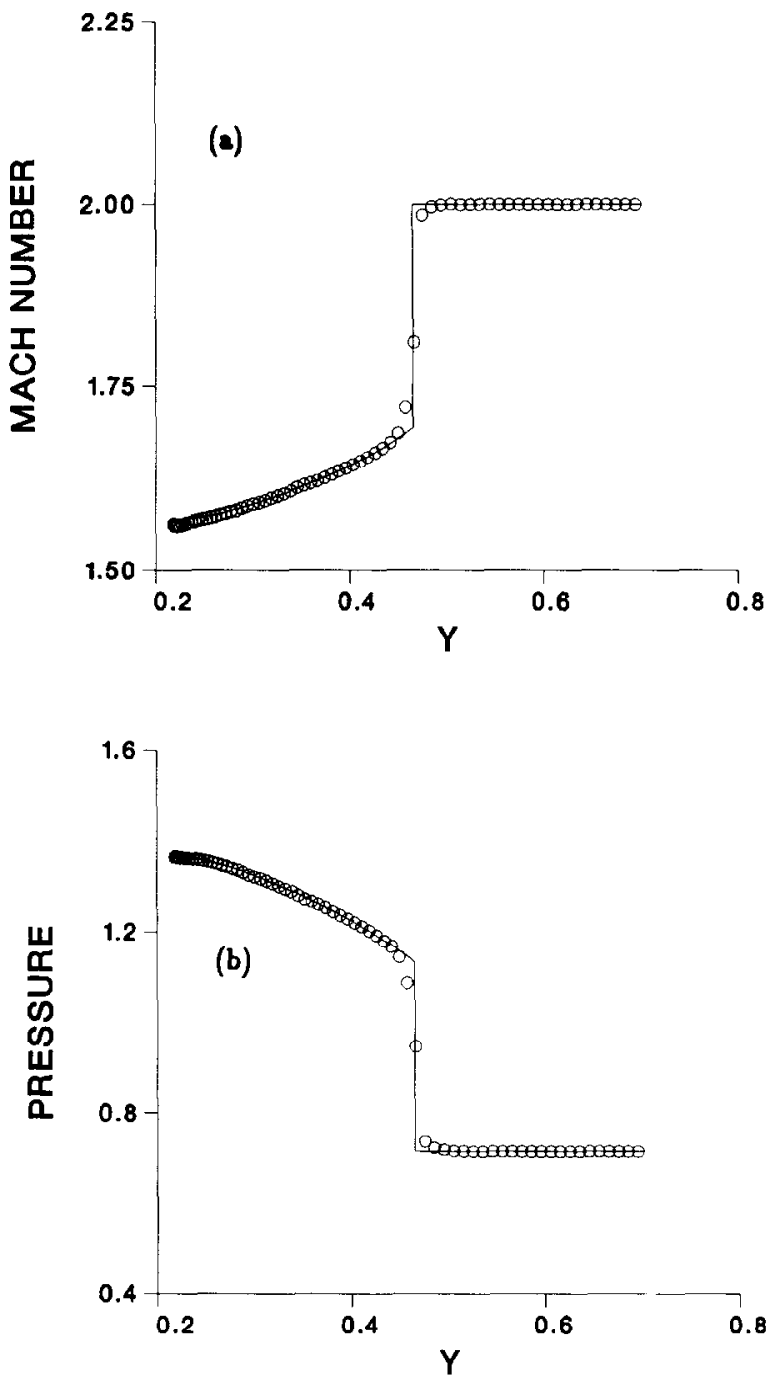

Fig. 17. Supersonic flow around an axisymmetric circular cone with $20^{\circ}$ cone angle $\left(M_{\infty}=2.0\right)$. Distribution of flow properties along a marching distance line $(x=0.6)$. (a) Mach number. (b) Pressure. 


\section{References}

[1] P.L. Roe. Characteristics-based upwind scheme for the Euler equations, Ann. Rev. Fluid Mech. 18 (1986) 337-365.

[2] G. Moretti, Computation of flows with shocks, Ann. Rev. Fluid Mech. 19 (1987) 313-337.

[3] S.K. Godunov, A difference method for numerical calculation of discontinuous solutions in hydrodynamics, Mat. Sb. 47 (1959) 271-306.

[4] H.M. Glaz and A.B. Wardlaw, A high-order Godunov scheme for steady supersonic gas dynamics, J. Comput. Phys. 58 (1985) 157-187.

[5] J. Glimm, Solution in the large for nonlinear hyperbolic systems of equations, Commun. Pure Appl. Math. 18 (1965) $697-715$.

[6] A.J. Chorin, Random choice solution of hyperbolic systems, J. Comput. Phys. 22 (1976) 517-536.

[7] G. Marshall and B. Plohr, A random choice method for two-dimensional steady supersonic shock wave diffraction problems, J. Comput. Phys. 56 (1984) 410-427.

[8] M. Pandolf, Computation of steady supersonic flows by a flux difference splitting method, Comput. Fluids 13(1) (1985) $37-46$.

[9] W.H. Hui and H.J. Van Roessel, Unsteady three-dimensional flow theory via material functions, in: NATO AGARD Symposium on Unsteady Aerodynamics-Fundamentals and Application to Aircraft Dynamics, CP-386, 1985, paper S1.

[10] C.Y. Loh and W.H. Hui, A new Lagrangian method for steady supersonic flow computation. I. Godunov scheme, J. Comput. Phys. 89 (1990) 207-240.

[11] W.H. Hui and C.Y. Loh, A new Lagrangian method for steady supersonic flow computation. II. Slip-line resolution, J. Comput. Phys. 103 (1992) 450-464.

[12] C.Y. Loh and W.H. Hui, A new Lagrangian random choice method for two-dimensional steady supersonic/hypersonic flow, AIAA J. 31 (1993) 2193-2194.

[13] B. van Leer, Towards the ultimate conservative difference scheme. V. Second order sequel to Godunov's method, J. Comput. Phys. 32 (1979) 101-136.

[14] A. Harten and S. Osher, Uniformly second-order essentially nonoscillatory schemes, I, SIAM J. Numer. Anal. 24(2) (1987) $279-309$.

[15] A. Harten, High resolution schemes for hyperbolic conservation laws, J. Comput. Phys. 49 (1983) $357-393$.

[16] A. Ferri, Elements of aerodynamics of supersonic flows (The Macmillan Company, New York, 1949) 153.

[17] N.H. Johannesen, Experiments on two-dimensional supersonic flow in comers and over concave surfaces, Philos. Mag. 43 (1952) 568-580.

[18] C.Y. Loh and M.-S. Liou, A new Lagrangian method for three-dimensional steady supersonic flow, J. Comput. Phys. 113 (1994) 224. 